For three component systems we may make use of the triangular diagrams introduced by Gibbs, which have been used extensively for temperature and composition diagrams of ternary alloys by Tammann and others. These diagrams are equilateral triangles, the corners represent pure substances, the sides mixtures of two components, and a point inside the triangle mixtures of three components.

Roozeboom and others have used such diagrams ruled with lines drawn parallel to the sides of the triangle. Thus in Fig. 3, a point, $\mathrm{P}$, denotes a mixture of $\mathrm{OC} \%$ of $\mathrm{A}, \mathrm{AR} \%$ of $\mathrm{B}, \mathrm{AQ} \%$ of $\mathrm{C}$.

It is self-evident that lines can be drawn through the area of the triangle, each point representing mixtures having the same density. For a percentage composition by volume and density diagram it is necessary to proceed as in the two component systems. Densities may then be read off directly.

Equation 3 may be written as follows:

$$
\mathrm{M} / \mathrm{D}=\Sigma\left(m_{a} / d_{a}, m_{b} / d_{b}, m_{r} / d_{c} \ldots \ldots \ldots m_{x} / d_{x}\right)
$$

from which $\mathrm{D}$ may be computed for any number of components.

Using this method, the densities for ointments were calculated. The necessary data were taken from the U. S. P., U. S. Dispensatory and "Landolt and Boernstein Tabellen."

\begin{tabular}{|c|c|c|c|}
\hline \multicolumn{4}{|c|}{ DENSITY OF OINTMENTS. } \\
\hline Unguentum Acidi Borici & 0.86 & Unguentum Aquae Rosae & 0.94 \\
\hline Unguentum Hydrarg. Amm. & 0.90 & Unguentum Sulphuris.... & 1.00 \\
\hline Unguentum Hydrarg Flav. . & 0.97 & Unguentum Zinci Oxidi. & 1.23 \\
\hline Unguentum Hydrarg. Dil.. . & 1.12 & Unguentum Belladonnae. & 0.95 \\
\hline Unguentum Hydrargyrum. & 1.76 & Enguentum Strammonii. & 0.95 \\
\hline Unguentum Iodi......... & 1.03 & Unguentum Gallae..... & .93 \\
\hline Unguentum Iodoformi. & 1.01 & Cnguentum Acidi Tannici. & 95 \\
\hline inguentum Phenolis. . & 0.94 & Unguentum Chrysarobini & .93 \\
\hline nowentum & 0.93 & Unguentum Picis Liquidi. & 99 \\
\hline
\end{tabular}

In addition to the references given in this text the following sources have been used in compiling the data, "Hagers Handbuch," "Chemiker Kalender," "Olsen's Annual" and the YFAR Book.

SUMMARY.

Methods have been described to compute the densities of ointments and the approximate densities of nearly all official ointments have been calculated.

The density of ammoniated mercury has been determined to be 5.63 at the temperature $27^{\circ} / 4^{\circ} \mathrm{C}$.

LONG ISI,AND CITY, N. Y.

\title{
THE PREPARATION OF BENZYL BENZOATE OF HIGH PURITY.
}

BY W. F. KAMM AND A. O. MATTHEWS.

Benzyl benzoate has been proposed as an antispasmodic and although it is still too early to determine to what extent it is actually efficient and to what extent we may be misled by the natural enthusiasm for a new therapeutic agent, it nevertheless seemed of importance to investigate the various methods of preparation of this compound and to examine also the specifications for purity that are accepted at the present time. 
Although benzyl benzoate is a simple organic compound which should be available in pure condition, it proved a surprising fact that the trade specimens examined and reported upon by the A. M. A. Laboratory in $1919^{1}$ were found to be distinctly impure. From among five specimens examined, one was found to contain chlorine, whereas all of them were found to be low in ester content, the variation being from $87.3 \%$ to $97.3 \%$. The standards adopted ${ }^{2}$ included the requirement of an ester content of not less than $95 \%$. The writers felt certain, however, that a product of higher purity might be produced even as a technical product, and accordingly the present work was undertaken.

The non-saponifiable impurities in benzyl benzoate may not prove to be very harmful, but very little information is available on this point. A pharmacological study of benzyl benzoate has recently been reported by Mason and Pieck $^{3}$ who found various commercial preparations to differ somewhat in toxicity. There is a possibility that these variations may be due to the non-ester impurities. On the other hand, the presence of chlorine is quite certain to indicate the presence of toxic benzyl chloride. ${ }^{4}$ It seemed of interest also to determine whether or not the impurities in benzyl benzoate are partly responsible for the disagreeable taste of the product.

Benzyl benzoate has been identified in certain natural plant products. ${ }^{5}$ In the laboratory, it has been prepared by the action of $(a)$ benzoyl chloride upon benzyl alcohol, $(b)$ benzyl chloride upon sodium benzoate, and (c) alcoholates upon benzaldehyde. ${ }^{6}$ Gomberg and Buchler ${ }^{7}$ have recently shown that reaction $b$ may be conducted even with aqueous solutions of sodium benzoate.

The Claisen method $(c)$ furnishes one of the most convenient and practical methods for the preparation of this ester. The materials involved are cheap, the yields are usually good, and, above all, no trouble need be anticipated from the presence of benzyl chloride if a good grade of benzaldehyde is used.

In the preparation of a product for therapeutic use, the last consideration is especially important and there is little doubt but that the bulk of benzyl benzoate now offered for sale is manufactured by this method. But why is the product impure?

According to Claisen ${ }^{8}$ benzyl benzoate is prepared in the following manner:

A 1.5-gram portion of sodium is dissolved in an exactly sufficient quantity of benzyl alcohol, 200 grams of benzaldehyde are added, and the mixture heated on the water-bath during several days. The reaction mixture is treated with $10 \mathrm{cc}$ of glacial acetic acid and with water, the oily layer being separated and subjected to fractional distillation. A yield of 150 grams of pure, constant boiling benzyl benzoate is obtained together with 40 grams of unchanged benzaldehyde.

These directions appear to be specific and simple enough to serve as a fairly satisfactory method of preparation, but when the experiment is conducted accord-

1 “Rep. Lab. Am. Med. Assoc.," 12, 84-7, 1919; C. A., 14, 3500, 1920.

2 "New \& Non-Official Remedies," 1920, 1921 and 1922.

${ }^{3} \mathrm{Jl}$. Lab. \& Clin., Med. 6, 1920.

4 J. Am. Chem. Soc., 42, 2061, 1920.

5 Ann., 152, 131, 1869.

${ }^{6}$ Claisen, Ber., 20, 649, 1887; cf. also J. Chem. Soc., 75, 1155, 1899.

7 J. Am. Chem. Soc., 42, 2059, 1920.

${ }^{8}$ Loc. cit., p. 649 . 
ing to directions, the results obtained are by no means constant. Although Claisen reports a yield of only $75 \%$, it is found that the method is capable at times of producing a $90-95 \%$ yield, while at other times the yield may be as low as $25 \%$. Moreover, the product obtained is not pure benzyl benzoate for it contains from $3 \%$ to $15 \%$ of unsaponifiable matter. These analytical results check well with the variation in ester content of the various samples examined by the American Medical Association Laboratory. ${ }^{1}$ It was the purpose of this investigation to explain the variable results obtained by the use of the Claisen method and to present laboratory directions that will result in a uniform high yield of a product possessing an ester content of $99 \%$ to $100 \%$. It is believed that this has been accomplished.

The low ester value of technical benzyl benzoate is not due to the presence of benzaldehyde as an impurity, in fact benzaldehyde will require an amount of alkali for saponification that is exactly equal to an equal weight of ester as is shown by the following equation:

\section{$2 \mathrm{C}_{6} \mathrm{H}_{5} \mathrm{CHO} \stackrel{\mathrm{NaOH}}{\longrightarrow} \quad \mathrm{C}_{6} \mathrm{H}_{5} \mathrm{CO}_{2} \mathrm{Na}+\mathrm{C}_{6} \mathrm{H}_{5} \mathrm{CH}_{2} \mathrm{OH}$}

This reaction of benzaldehyde must be kept in mind in proposing standards of purity for benzyl benzoate.

Reinvestigation of the Claisen method shows that variable results have been produced by the neglect of a few essential details in the manipulation. (a) Benzaldehyde is added to the solution of sodium in benzyl alcohol, whereas the reverse order should be used in mixing the two solutions. (b) Nothing is specified concerning the temperature of the sodium benzylate mixture at the time of mixing. It should be kept cold. (c) The final reaction should be conducted at a temperature slightly below that of the steam bath, and is complete in an hour; heating the mixture during several days is detrimental.

THE PREPARATION OF BENZYL, BENZOATE.

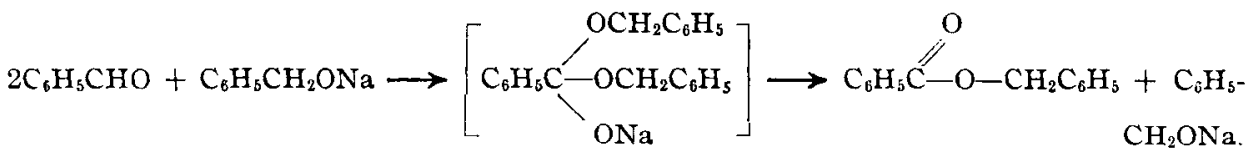

Three grams of metallic sodium are dissolved in 70 grams benzyl alcohol and after the solution has cooled to room temperature it is gradually added with thorough mixing, to $4 \tilde{\partial} 4$ grams of $\mathrm{C}$. $\mathrm{P}$. benzaldehyde (which must contain less than one percent of benzoic acid). The reaction mixture has a tendency to become warm but the temperature should be kept slightly below $50-60^{\circ}$ by cooling if necessary. A pasty gelatinous mass results which, after about one-half hour when the mixture no longer shows evidence of generating heat spontaneously, is warmed on the water-bath during about an hour with occasional shaking.

The cooled reaction product is treated with $200 \mathrm{cc}$ of water, the layer of oil separated, washed once with a second portion of water, and subjected to vacuum distillation. The first fraction of the distillate will contain benzyl alcohol together with unchanged aldehyde, as well as a small quantity of water. The tempera-

1 Loc. cit. 
ture will then rise rapidly to the boiling point of benzyl benzoate when the receivers are changed. The product boils at $1,44-5^{\circ}$ (uncorrrected) at $15 \mathrm{~mm}$. and analysis by saponification shows it to consist of $99 \%$, ester. A yield of $+10-420$ grams is obtained which corresonds to $901-933 \%$ of theory. 'This benzyl benzoate supercools readily but after solidifying melts within one degree of the highest recorded value $\left(19.4^{\circ}\right)$ and therefore need not be refractionated unless material of exceptional grade is required.

\section{NOTES.}

In the presence of sodium benzylate, two molecules of benzaldehyde react with the alcoholate to form the addition product indicated in the equation written above. The addition product then decomposes in the manner indicated. When the reaction mixture is overheated an important side-reaction may occur.

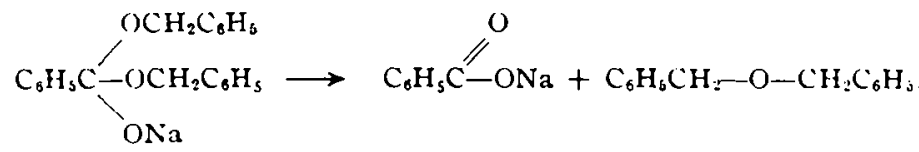

Dibenzyl ether no doubt forms the chief impurity in benzyl benzoate. Since the boiling point of the former lies near that of the ester, it is not removed during the process of purification by distillation.

We may now explain the causes of variations in yield by the use of the older methods. When benzaldehyde is added to the alcoholate, and especially when the latter is still warm, local overheating results; in fact, the temperature may rise far above $100^{\circ}$ with the result that benzyl ether is formed. Simultancously, the sodium benzylate is converted into sodium benzoate, which is of no value for inducing the desired reaction and consequently very little benzyl benzoate is obtained. The same side-reactions explain the failure of this experiment when the benzyl alcohol used in preparing the catalyst (sodium benzylate) is contaminated with benzaldehyde.

The benzyl alcohol used in this preparation must be free from aldehyde. One cc dissolved in $50 \mathrm{cc}$ of water and treated with a freshly prepared clear solution of phenyl hydrazine acetate should give no appreciable precipitate.

The benzaldehyde should be titrated in order to determine its acidity. If it contains sufficient benzoic acid to react with a considerable proportion of the sodium alcoholate, a poor yield of ester will be obtained. Less than one percent of benzoic acid will not interfere seriously with the yields obtained, but the presence of larger quantities of the acid will be found to be detrimental.

The order of mixing the reagents and the temperature of the ingredients at the time of mixing is the most important factor in the experiment. The temperature at which the reaction-mixture is maintained after mixing, provided that it is held below $100^{\circ}$, is less important from the standpoint of purity.

When benzaldehyde was treated with a cold solution of sodium benzylate and the reaction-mixture maintained at steam-bath temperature for about two days, a $90 \%$ yield of product was obtained. This product on analysis was found to consist of $97 \%$ ester. Benzyl alcohol prepared from it did not dissolve to give a clear $2 \%$ aqueous solution. In a reaction in which the benzaldehyde was added to the benzylate without cooling, there was obtained a low yield $(20-30 \%)$ of a product 
possessing a very low ester value. According to the improved method now presented, a product is obtained which analyzes $99-100 \%$ ester.

The reaction mixture is not treated with acetic acid as usually recommended for the reason that such a procedure yields an ester contaminated with benzoic acid.

The recovered benzyl alcohol can be used for the preparation of a second lot of benzyl benzoate, only after it has been boiling with strong caustic to remove any benzaldehyde present.

The benzyl benzoate of special purity described above was found to possess a somewhat improved taste. Unfortunately, however, the extremely unpleasant after-taste that handicaps benzyl benzoate appears to be typical of the pure substance, since it is alleviated only partly by removal of the main impurity.

CALCIUM CHLORIDE ADDITION COMPOUND OF BENZYL BENZOATE.

As has been shown by $\mathrm{J}$. Allain ${ }^{1}$ ethyl acetate is capable of forming addition compounds with inorganic salts such as $\mathrm{MgCl}_{2}$ and $\mathrm{CaCl}_{2}$ in which one molecule of the inorganic salt is combined with 2 molecules of the acetate. A similar class of compounds is obtained if benzyl benzoate is allowed to react with anhydrous $\mathrm{CaCl}_{2}$.

Varying amounts of benzyl benzoate were added to $1 / 4$ molecular portions of pulverized fused calcium chloride. The flasks were corked and allowed to stand 24 hours. Solidification of the contents took place after $3-4$ hours, but no evolution of heat was noted.

The reaction products were covered with ether, the lumps broken up with a stirring rod and the solid filtered and washed several times with ether. The ether used had been previously dried over $\mathrm{CaCl}_{2}$. The filtered solid was further freed from adhering ether by exposure to dry air during 20-30 minutes. The combined ether washings were evaporated on the water-bath and the recovered benzyl benzoate weighed. From three experiments the following results were obtained:

$\begin{array}{cccc}\text { Benzy benzoate } & \text { Fused CaCl} & \text { Benzoate } \\ \text { Gm. } & \begin{array}{c}\text { Gm. } \\ \text { recovered } \\ \text { Gm. }\end{array} & \begin{array}{c}\text { Wt. of addition } \\ \text { product Gm. }\end{array} \\ 42 & 28 & 18 & 45 \\ 63 & 28 & 38 & 50 \\ 84 & 28 & 62 & 46\end{array}$

The amount of benzyl benzoate reacting with a given weight of calcium chloride is found to be independent of the amount of ester initially added. This is shown not merely by the weight of the calcium chloride addition product formed but also by the amount of ester recovered.

The calcium chloride addition product is a white, crystalline powder which slowly absorbs moisture when exposed to the atmosphere and therefore must be preserved in tightly corked containers. From the proportions of the two constituents involved in the reaction, the composition of the addition compound is found to correspond to the formula, $\mathrm{C}_{14} \mathrm{H}_{12} \mathrm{O}_{2} .2 \mathrm{CaCl}_{2}$.

PAPER No. 5 ,

Chemical Research Dept.,

Parke, Davis \& Company.

'J., 1885, 1159. 